\title{
A cross-layer switching of OFDMA and MU-MIMO for future WLAN systems
}

\author{
Tomoki Murakami ${ }^{1 \text { a) }}$, Yasushi Takatori ${ }^{1}$, Masato Mizoguchi ${ }^{1}$, \\ and Fumiaki Maehara ${ }^{2}$ \\ ${ }^{1}$ NTT Network Innovation Laboratories, Nippon Telegraph and Telephone \\ Corporation, 1-1 Hikarinooka, Yokosuka, Kanagawa 239-0847, Japan \\ ${ }^{2}$ Graduate School of Fundamental Science and Engineering, Waseda University, \\ 3-4-1 Ookubo, Shinjuku, Tokyo 169-8555, Japan
}

a)murakami.tomoki@lab.ntt.co.jp

\begin{abstract}
We propose a cross-layer switching method of orthogonal frequency division multiple access (OFDMA) and multiuser multiple input multiple output (MU-MIMO) for the future wireless local area network systems. The proposed method, employed on the medium access control layer, switches between OFDMA and MU-MIMO as the transmission method after processing by using physical layer information such as an overhead of channel state information feedback, station number, and data length in order to improve the transmission efficiency. Simulation results show that the proposed method achieves higher total throughput than conventional OFDMA or MU-MIMO where switching is not performed.
\end{abstract}

Keywords: multiuser multiple input multiple output (MU-MIMO), orthogonal frequency division multiple access (OFDMA), cross-layer, wireless LAN system

Classification: Wireless Communication Technologies

\section{References}

[1] 3GPP TS 36.913, "Evolved universal terrestrial radio access (E-UTRA); Requirements for further advancements for EUTRA LTE-Advanced."

[2] IEEE Std. 802.11ac-2013, IEEE SA, Dec. 2013.

[3] Q. H. Spencer, C. B. Peel, A. L. Swindlehurst, and M. Haardt, "An introduction to the multi-user MIMO downlink," IEEE Commun. Mag., vol. 42, no. 10, pp. 6067, Oct. 2004. DOI:10.1109/MCOM.2004.1341262

[4] R. Kudo, Y. Takatori, K. Nishimori, A. Ohta, S. Kubota, and M. Mizoguchi, "A new user selection measure in block diagonalization algorithm for multiuser systems," IEICE Trans. Commun., vol. E92-B, no. 10, pp. 3206-3218, Oct. 2009. DOI:10. 1587/transcom.E92.B.3206

[5] C. Y. Wong, R. S. Cheng, K. B. Letaief, and R. D. Murch, "Multiuser OFDM with adaptive sub-carrier, bit, and power allocation," IEEE J. Sel. Areas Comm., vol. 17, no. 10 , pp. $1747-1758$, Oct. 1999. DOI:10.1109/49.793310

[6] A. Doufexi and S. Armour, "Design considerations and physical layer performance results for a 4G OFDMA system employing dynamic subcarrier allocation," Proc. IEEE PIMRC, vol. 1, pp. 357-361, Sep. 2005. DOI:10.1109/PIMRC.2005. 1651458 
[7] C. Antron-Haro, O. Svedman, M. Bengtsson, A. Alexiou, and A. Gameiro, "Crosslayer scheduling for multi-user MIMO systems," IEEE Commun. Mag., vol. 44, no. 9, pp. 39-45, Sep. 2006. DOI:10.1109/MCOM.2006.1705977

[8] N. Mokari, M. R. Javan, and K. Navaie, "Cross-layer resource allocation in OFDMA systems for heterogeneous traffic with imperfect CSI," IEEE Trans. Vehicular Technol., vol. 59, no. 2, pp. 1011-1017, Feb. 2010. DOI:10.1109/TVT. 2009.2035131

[9] M. Bohge, J. Gross, and A. Wolisz, "Dynamic resource allocation in OFDM systems: an overview of cross-layer optimization principles and techniques," IEEE Netw., vol. 21, no. 1, pp. 53-59, Feb. 2007. DOI:10.1109/MNET.2007.314539

\section{Introduction}

For high-speed transmission, MIMO and carrier aggregation (CA) have been adopted by wireless systems such as LTE and WLAN $[1,2]$. However, assuming compact and low power consumption stations (STAs), the STA generally has fewer antennas and less frequency bandwidth than the access point (AP). Therefore, transmission efficiency degrades because AP cannot fully utilize the spatial streams and frequency bandwidth at the same time.

To improve transmission efficiency, research has focused on two technologies: multiuser MIMO (MU-MIMO) [3, 4] and OFDMA [5, 6]. MU-MIMO achieves high transmission efficiency even if each STA has a single antenna. OFDMA is also effective even if each STA has narrow frequency bandwidth. On the physical (PHY) layer, the channel capacity of MU-MIMO is higher than that of OFDMA because AP transmits spatial streams at same time in the same frequency bandwidth. However, MU-MIMO demands that STAs feed channel state information (CSI) back to the AP to calculate beamforming precoding weight. Therefore, the total throughput on the medium access control (MAC) layer is degraded by the overhead of CSI feedback. In OFDMA, the throughput degradation is small because it dispenses with CSI feedback. Actual wireless systems need a crosslayer method, which considers both the transmission speed on the PHY layer and the transmission efficiency on the MAC layer.

It is becoming more and more necessary to use the cross-layer approach when implementing protocols $[7,8]$ to improve transmission efficiency. Conventional methods target channel capacity maximization on the PHY layer by the adaptive allocation of user combination, subcarrier, bit, and power allocation according to characteristic of the different packets and CSI. In the realistic environment, it is necessary to maximize not only the channel capacity but also the throughput so the overhead of CSI feedback must be considered. However, no study has evaluated cross-layer method with such overhead.

This paper proposes a cross-layer switching of OFDMA and MU-MIMO for the future WLAN systems. The proposal, which resides on the MAC layer, selects OFDMA or MU-MIMO given access to PHY information such as overhead of CSI feedback, STA number, and data length. In MU-MIMO, AP uses the precoding weight calculated by the zero-forcing (ZF) method. In OFDMA, AP transmits STAs without beamforming by using the allocation of $20 \mathrm{MHz}$ channels to ensure 
backward compatibility with conventional WLAN systems. Simulation results show that the proposal achieves higher total throughput than conventional OFDMA or MU-MIMO where switching is not performed.

\section{System model}

We consider OFDM system where AP has $N$ antennas for communication with $M$ STAs equipped with a single antenna. It is assumed that signaling complies with the frame format of IEEE 802.11ac [2].

Fig. 1(a) shows the OFDMA frame sequence. OFDMA does not use both beamforming or dynamic resource allocation to avoid the CSI feedback overhead [9]. We also assume that equal power is allocated to each subcarrier and each STA to equalize communication quality. Moreover, we assume the frequency allocation of $20 \mathrm{MHz}$ bandwidth to ensure backward compatibility with conventional WLAN systems. If AP uses $80 \mathrm{MHz}$ bandwidth, AP transmits to four STAs with $20 \mathrm{MHz}$ bandwidth or two STAs with $40 \mathrm{MHz}$ bandwidth. The frame sequence consists of data, block acknowledgement (BA), and BA request (BAR) frames. In the sequence, after AP carries out carrier sense between distributed inter-frame space (DIFS), waits for back off time expiry, and transmits the data frame to multiple STAs by OFDMA without beamforming. AP and STAs then check for errors by BA and BAR under time division multiple access.

Fig. 1(b) shows the MU-MIMO frame sequence. In MU-MIMO, we also assume that equal power is allocated to each STA. The beamforming precoding weight is calculated by ZF, which is a low-complexity weight calculation method. The frame sequence consists of null data packet announcement (NDPA), NDP, CSI feedback (CSI-FB), beamforming report poll (BRP), data, BA and BAR frames. In the sequence, AP broadcasts NDPA and NDP frames to STAs for CSI estimation, each STA estimates CSI from the received NDP frame and feeds CSI back to AP via CSI-FB. We assume unitary matrix compression feedback; it feeds CSI back in the form of angles representing the compressed beamforming feedback matrices per subcarriers, as supported by IEEE802.11ac [2]. In CSI-FB frame, we assume that the bit amounts for the compressed beamforming report field $\psi$ and $\theta$,

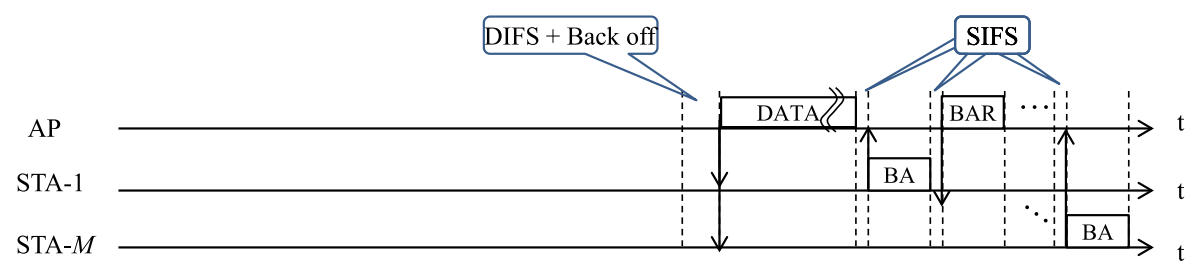

(a). OFDMA frame sequence

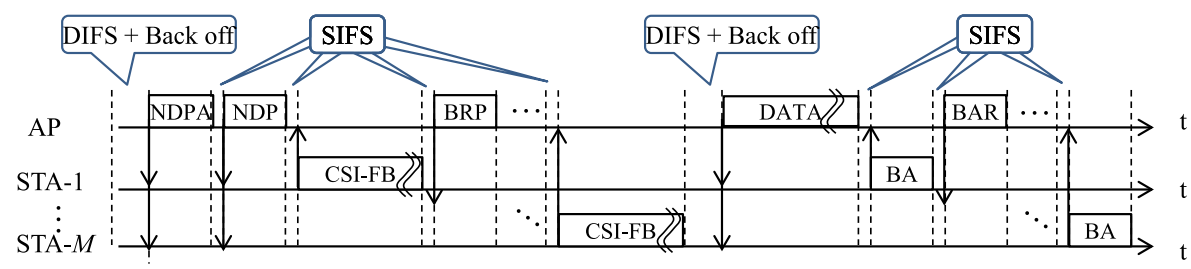

(b). $\quad$ MU-MIMO frame sequence

Fig. 1. Frame sequences 
are set to 7 and 9 bits, respectively. AP lets other STAs feed CSI back via BRP. After receiving CSI, AP calculates the precoding weight for that STA. AP then transmits the data frames to the STAs. Finally, AP and STA check for errors by BA and BAR.

\section{Cross-layer switching of MU-MIMO and OFDMA}

This section indicates the cross-layer switching of OFDMA and MU-MIMO. The proposal is set on the MAC layer, and selects OFDMA or MU-MIMO after processing PHY information such as the overhead of CSI feedback, STA number, and data length. It is assumed that AP will use the optimal modulation and coding scheme (MCS) per STA in MU-MIMO or OFDMA, STA also will use the same MCS in MU-MIMO or OFDMA, and all STAs have the same data length. $R^{a}$ and $R^{b}$ are the total throughputs of OFDMA and MU-MIMO on the MAC layer and are expressed as

$$
R^{a}=\frac{T_{\text {data }}}{T_{\text {total }}^{a}} \sum_{n=1}^{N_{\text {sta }}} \frac{N_{\text {sub }, n}^{a} C_{n}^{a} \log _{2} M_{n}^{a}}{T_{S}}, \quad R^{b}=\frac{T_{\text {data }}}{T_{\text {total }}^{a}} \sum_{n=1}^{N_{\text {sta }}} \frac{N_{\text {sub }, n}^{b} C_{n}^{b} \log _{2} M_{n}^{b}}{T_{S}},
$$

where $T_{\text {data }}, T_{s}, T_{G I}, T_{\text {total }}^{a}$ and $T_{\text {total }}^{b}$ are the times of data except PHY and MAC headers, OFDM symbol (4 $\mu \mathrm{s})$, guard interval ( $800 \mathrm{~ns})$, OFDMA sequence, and MU-MIMO sequence, respectively. $N_{\text {sub, },}{ }^{a}, N_{\text {sub,n }}{ }^{b}, C_{n}{ }^{a}, C_{n}{ }^{b}, M_{n}{ }^{a}$ and $M_{n}{ }^{b}$ are the subcarrier number, the coding rate, and the bit number of modulation at the $n^{\text {th }}$ STA $\left(n=1, \ldots, N_{\text {sta }}\right) . T_{\text {total }}{ }^{a}$ is calculated by

$$
\begin{aligned}
T_{\text {total }}^{a}= & T_{D I F S}+T_{B O}+\left(T_{\text {header }}+T_{\text {data }}\right)+T_{\text {SIFS }}+T_{B A} \\
& +\left(N_{\text {sta }}-1\right)\left(2 T_{\text {SIFS }}+T_{B A R}+T_{B A}\right),
\end{aligned}
$$

where $T_{D I F S}, T_{B O}, T_{\text {header }}, T_{S I F S}, T_{B A}$, and $T_{B A R}$ are the times of DIFS $(34 \mu \mathrm{s})$, backoff $(67.5 \mu \mathrm{s})$, PHY and MAC headers $(44 \sim 88 \mu \mathrm{s})$ based on IEEE 802.11ac [2], short inter-frame space (SIFS, $16 \mu \mathrm{s}), \mathrm{BA}(44 \sim 88 \mu \mathrm{s})$, and BAR $(44 \sim 76 \mu \mathrm{s})$, respectively. In above parentheses, the frame length ranges according to MCS and bandwidth are described.

$$
\begin{aligned}
T_{\text {total }}^{b}= & T_{D I F S}+T_{B O}+T_{N D P A}+T_{N D P}+T_{S I F S}+T_{C S I} \\
& +\left(N_{\text {sta }}-1\right)\left(2 T_{S I F S}+T_{C S I}+T_{B R P}\right)+T_{D I F S}+T_{B O}+\left(T_{\text {header }}+T_{\text {data }}\right), \\
& +T_{S I F S}+T_{B A}+\left(N_{\text {sta }}-1\right)\left(2 T_{S I F S}+T_{B A R}+T_{B A}\right)
\end{aligned}
$$

where $T_{N D P A}, T_{N D P}, T_{C S I}$, and $T_{B R P}$ are the times of NDPA $(44 \sim 52 \mu \mathrm{s})$, NDP $(40 \sim 52 \mu \mathrm{s})$, CSI-FB $(120 \sim 1100 \mu \mathrm{s})$, and BRP $(44 \sim 48 \mu \mathrm{s})$, respectively. In the proposal, AP calculates the total throughputs of OFDMA and MU-MIMO by (2) and (3). AP then uses the transmission method that offers the higher total throughput to transmit to STAs.

\section{Simulation results}

This section is compared OFDMA and MU-MIMO performances with the overhead of CSI feedback and the proposal performance. In this simulation, the center frequency and bandwidth are $5.2 \mathrm{GHz}$ and $80 \mathrm{MHz}$, respectively. The number of subcarriers (bandwidth) is 52 (20 MHz), 108 (40 MHz), and 234 (80 MHz). In MU- 
MIMO, all STAs receive on the $80 \mathrm{MHz}$ bandwidth, or some fraction thereof depending STA number; $20 \mathrm{MHz}$ bandwidth for four STAs and $40 \mathrm{MHz}$ bandwidth for two STAs in OFDMA. AP has four antennas to communicate with up to four STAs, each equipped with a single antenna. Then we examine signal to noise power ratios (SNR) of 25,30 , or $35 \mathrm{~dB}$; propagation channels exhibit independent and identically distributed MIMO static Rayleigh fading. AP and STA support MCS of IEEE 802.11ac, and select MCS whose packet error rate at the cumulative distribution function (CDF) $10 \%$ is less than 0.1 .

Fig. 2 shows the total throughput with overhead on the MAC layer offered by OFDMA or MU-MIMO for SNRs of 25, 30, and $35 \mathrm{~dB}$. The total throughput indicates the summation of STA throughputs. In Fig. 2(a), AP with four antennas transmits the data lengths of $0.5,1.0$ and $2.0 \mathrm{~ms}$ to two or four STAs. When STA number, $N_{s t a}$, is two, MU-MIMO offers higher total throughput than OFDMA regardless of SNR and data length. There are two reasons of these results. One is that the overhead of CSI feedback is small compared to data length because AP transmits to only two STAs. Another is that AP can transmit to STAs by using higher MCS because of beamforming array gain. Since this environment has more $\mathrm{AP}$ antennas than stream number, the degree of freedom (DoF) provided by MIMO realizes beamforming array gain. However, setting $N_{s t a}=4$ yields different characteristics. At high SNR (35 dB), MU-MIMO has higher total throughput than OFDMA regardless of data length. However, OFDMA performance exceeds MUMIMO performance at low SNR $(25 \mathrm{~dB})$. This is because transmission efficiency is degraded by the overhead increase generated by the larger STA number. And, since DoF cannot be used to secure beamforming array gain because AP antenna number equals STA number, it is difficult to allocate higher MCS. Finally, at medium SNR $(30 \mathrm{~dB})$, superiority depends on data length. From the above results, we found that MU-MIMO performance is better than OFDMA performance if there is some DoF margins as shown in the results of $N_{\text {sta }}=2$ in Fig. 2. When there is no DoF margin $\left(N_{s t a}=4\right)$, the relative superiority of OFDMA and MU-MIMO switches depending on data length or SNR.

Fig. 3(a) shows the total throughput with OFDMA, MU-MIMO, and the proposal versus data length. And Fig. 3(b) shows the throughput gain from the proposal to OFDMA or MU-MIMO. In these figures, we assume that $\mathrm{SNR}=30 \mathrm{~dB}$ and $N_{s t a}=4$. As can be seen, the proposal achieves higher total throughput than conventional OFDMA or MU-MIMO where switching is not performed. For example, we found that there is 1.33 gains in short length packet of $0.5 \mathrm{~ms}$, and

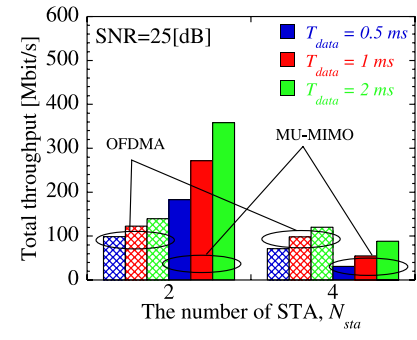

(a) $\mathrm{SNR}=25 \mathrm{~dB}$

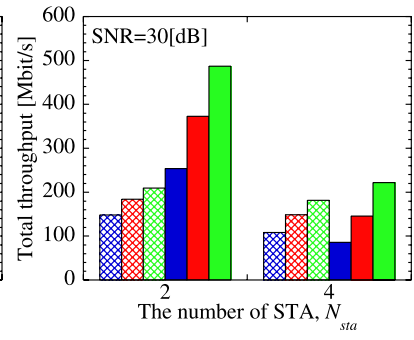

(b) $\mathrm{SNR}=30 \mathrm{~dB}$

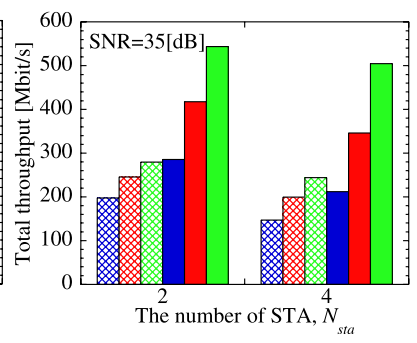

(c) $\mathrm{SNR}=35 \mathrm{~dB}$

Fig. 2. Total throughput versus STA number 


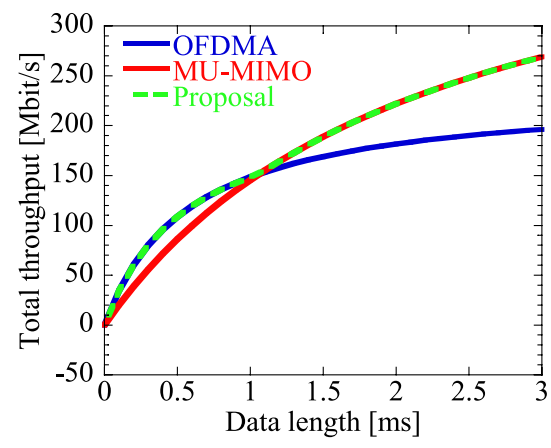

(a) Total throughput

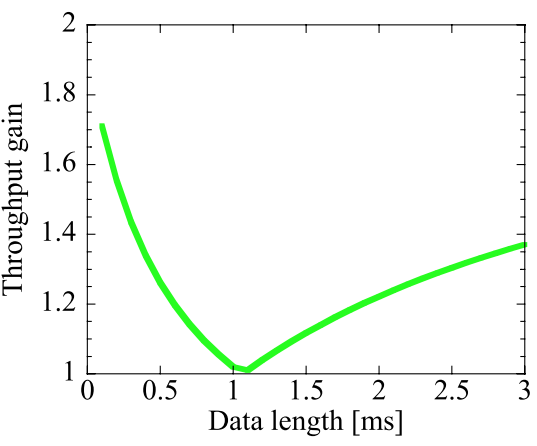

(b) Throughput gain

Fig. 3. Total throughput and gain versus data length

there is 1.37 gains in long length packet of $3 \mathrm{~ms}$. Therefore, these results clarify that the transmission performance of future wireless systems can be improved by switching between OFDMA and MU-MIMO.

\section{Conclusion}

We propose the cross-layer switching which is set on the MAC layer, and selects OFDMA or MU-MIMO given PHY information such as the overhead of CSI feedback, STA number, and data length. Results showed that MU-MIMO performance is better than OFDMA performance if DoF provides some margins. However, the proposed method achieves higher total throughput than conventional OFDMA or MU-MIMO where switching is not performed when DoF provides no margin. In this simulation, since we assume that static fading and OFDMA without CSI, MUMIMO is advantageous. Therefore, we expect that the proposal will become more effective in realistic environment. 